\title{
Reconstruction methods during laparoscopic gastrectomy
}

\author{
Amilcare Parisi ${ }^{1}$, Chang-Ming Huang ${ }^{2,3,4}$ \\ ${ }^{1}$ St. Mary's Hospital, Department of Digestive Surgery, Terni, Italy. \\ ${ }^{2}$ Department of Gastric Surgery, Fujian Medical University Union Hospital, Fuzhou, Fujian Province, China. \\ ${ }^{3}$ Department of General Surgery, Fujian Medical University Union Hospital, Fuzhou, Fujian Province, China. \\ ${ }^{4}$ Key Laboratory of Ministry of Education of Gastrointestinal Cancer, Fujian Medical University, Fuzhou, China.
}

To Cite

Parisi A, Huang C-M. Reconstruction methods during laparoscopic gastrectomy. J Gastric Surg 2019; 1(1): 19-21

\section{Pubblication history}

Received: October 29, 2019

Revised: November 20, 2019

Accepted: December 2, 2019

Article in press: December 10, 2019

Published online: December 16, 2019

\section{Correspondence to}

Dr. Amilcare Parisi

Department of Digestive Surgery,

St Mary's Hospital, Terni 05100, Italy

amilcareparisi@virgilio.it

Telephon: +393387312313

\begin{abstract}
Laparoscopic surgery has been progressively developed in gastric cancer surgery. Although the feasibility of laparoscopic procedures has been demonstrated, there are many issues being debated, including the feasibility of intracorporeal anastomoses. The anastomosis execution method has a major impact on perioperative outcomes. Few articles in the literature described a full laparoscopic execution of the reconstructive phase and others do not explained how this phase is run or mixed procedures were reported.

This article offers the reader detailed description of the most popular laparoscopic methods and how they are performed.
\end{abstract}




\section{Background}

Minimally invasive gastrectomy is gaining popularity worldwide as it is associated with earlier recovery of patients when compared with traditional open surgery[1-3].

Surgical outcomes related to emerging techniques for reconstruction deserve attention.

Laparoscopic gastrectomy has conventionally been followed by reconstruction through a mini-laparotomy. Recently, investigators have described numerous techniques for totally intracorporeal procedures to improve surgical efficiency and invasiveness.

Some investigators have shown quicker recovery of bowel function when compared with extracorporeal reconstructions[4].

Probably, this allows for less manipulation of the bowel and is useful particularly in obese patients where access through a minilaparotomy can be limited.

In this technical note we'll focus on the most interesting and newest strategies for reconstruction proposed for the laparoscopic approach.

\section{Laparoscopic Techniques}

"Laparoscopy-assisted gastrectomy" requires a minilaparotomy for specimen removal and extracorporeal anastomoses. This approach is still widely performed and applied in most clinical trials.

Intracorporeal anastomosis, which made this procedure the so called "totally laparoscopic surgery," enables more sophisticated reconstruction methods.

It can decrease the length of the mini-laparotomy and shift the incision for specimen retrieval in more convenient positions (below the umbilicus, McBurney incision), which results in reduced incision-related pain. After intracorporeal Billroth I was safely performed with delta-shaped anastomosis, totally laparoscopic distal subtotal gastrectomy gained popularity.

Many efforts have also been made to perform the intracorporeal esophago-jejunal anastomosis during total gastrectomy. While hand-sewn anastomosis is not an attractive choice in the laparoscopic approach, it is gaining attention by robotic surgeons[5].

\section{Reconstruction after distal gastrectomy}

Numerous procedures and technical solutions have been proposed for reconstruction during laparoscopic distal gastrectomy.

Gastro-duodenostomy is one of the most common types of reconstruction.

In literature[6], extracorporeal procedures are performed with the modified double stapling method or the posterior wall method, while the modified delta shaped method is the most attractive technique for the intracorporeal approach.

For the two extracorporeal methods, usually a $5 \mathrm{~cm}$ length of transverse mini-laparotomy is made on the right upper quadrant of the abdomen.

The Modified Delta-Shaped Method, as developed by Prof. Huang[7], is performed with the following described steps.

The stomach is resected from the greater curvature to the lesser curvature. Small incisions are created on the greater curvature of the remnant stomach and the anti- mesenteric side of the jejunum located $12-15 \mathrm{~cm}$ distal to the Treitz ligament. After the $60 \mathrm{~mm}$ endoscopic linear stapler is opened, one stapler limb is first inserted into the jejunum incision towards the direction of the jejunal proximal end. The jejunum is pulled out forward, and the other limb of stapler is inserted into the incision on the remnant stomach greater curvature. The stapler is fired to complete a side-to-side gastrojejunostomy with a common stab incision.

The common stab incision is closed using the $60 \mathrm{~mm}$ linear stapler. At the end we can clearly see the inverted " $\mathrm{T}$ " shaped appearance of the Modified Delta-Shaped Method anastomosis.

Other possible reconstruction is through a gastrojejunostomy.

Surgeons have described the Billroth II method or the Roux-en-Y method, both performed with the extracorporeal or the intracorporeal approach.

The Billroth II method is very fast and convenient. Small incisions are created on the greater curvature of the remnant stomach and the anti-mesenteric side of the jejunum. After the $60 \mathrm{~mm}$ endoscopic linear stapler is opened, one stapler limb is first inserted into the jejunum, while the other limb into the incision on the remnant stomach greater curvature. The stapler is fired to complete a side-to-side gastro-jejunostomy with a common stab incision. The latter is closed using the linear stapler.

In case of a Roux-en-Y extracorporeal reconstruction an upper midline or a left subcostal transverse skin incision is necessary[8].

A totally Roux-en-Y intracorporeal reconstruction [9] can be performed with the following described steps. A small opening is made at the end of the stapling line on the greater curvature side of the stomach and at the jejunum, $20 \mathrm{~cm}$ away from the Treitz ligament. Then the linear stapler is inserted and fired. The common entry hole is closed by the linear stapler. Next, in the same manner, the side to side jejuno-jejunostomy is performed at $25 \mathrm{~cm}$ below the gastro-jejunostomy using the linear stapler. Finally, a $45 \mathrm{~mm}$ knifeless linear stapler (uncut procedure) is applied to the afferent loop between the gastro-jejunostomy and jejuno-jejunostomy for preventing bile reflux.

\section{Reconstruction after total gastrectomy}

Laparoscopic esophago-jejunostomy is the most critical and technically challenging step during laparoscopic total gastrectomy. Various methods for esophagojejunostomy have been introduced, but no standard protocol exists.

Extracorporeal and intracorporeal anastomosis are described, and further classified into side-to-side anastomosis using linear staplers and end-to-side anastomosis when using circular staplers.

Usually, procedures involving the circular stapler need at least a $5 \mathrm{~cm}$ vertical mini-laparotomy in the epigastrium. It is possible to use a laparoscopic purse-string suture instrument, while other surgeons prefer the use of an anvil delivery device (OrVil) that is designed to insert the anvil trans-orally into the esophagus. The side-to-side jejunojejunostomy is usually performed 
extracorporeally using a linear stapler.

The Intracorporeal Isoperistaltic Jejunum-Later-Cut Overlap method performed by Prof. Huang $[6,7]$ can be performed with the following steps.

The esophagus is transected applying a linear stapler. Small incisions are made on the left side of the esophagectomy margin and on the boundary of the mesenteric membrane of the jejunum about $20 \mathrm{~cm}$ from the Treitz ligament. After each incision is inserted into one of the limbs of the stapler, the fork of the stapler is closed and fired to perform a side-to-side esophagojejunostomy.

Confirmation of no injury or bleeding is made via the common stab incision, which is then manually sutured. Finally, the lateral-lateral jejunojejunostomy is created at about $40-45 \mathrm{~cm}$ from the esophageal-jejunum anastomosis.

\section{Conclusion}

laparoscopy is a constantly evolving surgery and nowadays the development of new instruments and better viewing images allows the surgeon to safely deal with demanding operation steps, such as reconstruction after gastrectomy.

A completely intracorporeal approach appears to offer the patient significant benefits in the postoperative period and should be the approach of choice in minimally invasive surgery.

\section{Acknowledgements \\ Not applicable}

\section{Contributors}

$\mathrm{AP}, \mathrm{CMH}$ were involved in conception of the study, designing the study, analyzing the literature, references searching and writing the final manuscript.

\section{Funding}

None.

\section{Competing interests}

No benefits in any form have been received or will be received from a commercial party related directly or indirectly to the subject of this article.

\section{Availability of data and materials}

The datasets used and/or analyzed during the current study are available from the corresponding author on reasonable request.

\section{Ethics approval}

Not applicable

\section{Provenance and peer review}

Not commissioned; externally peer reviewed.

\section{Open access}

This is an Open Access article distributed in accordance with the Creative Commons Attribution NonCommercial (CC BY-NC 4.0) license, which permits others to distribute, remix, adapt, build upon this work noncommercially, and license their derivative works on different terms, provided the original work is properly cited and the use is non-commercial. See: http:// creativecommons.org/licenses/by-nc/4.0/

\section{References}

[1] Huang L, Li TJ. Laparoscopic surgery for gastric cancer: where are we now and where are we going? Expert Rev Anticancer Ther. 2018;18:1145-57.

[2] Desiderio J, Stewart CL, Sun V, Melstrom L, Warner S, Lee B, et al. Enhanced Recovery after Surgery for Gastric Cancer Patients Improves Clinical Outcomes at a US Cancer Center. J Gastric Cancer. 2018;18:230-41.

[3] Parisi A, Reim D, Borghi F, Nguyen NT, Qi F, Coratti A, et al. Minimally invasive surgery for gastric cancer: A comparison between robotic, laparoscopic and open surgery. World J Gastroenterol. 2017;23:2376-84.

[4] Milone M, Manigrasso M, Burati M, Elmore U, Gennarelli N, Cesare Giglio $\mathrm{M}$, et al. Intracorporeal versus extracorporeal anastomosis after laparoscopic gastrectomy for gastric cancer. A systematic review with meta-analysis. J Visc Surg. 2019;156:305-18.

[5] Parisi A, Ricci F, Gemini A, Trastulli S, Cirocchi R, Palazzini G, et al. New totally intracorporeal reconstructive approach after robotic total gastrectomy: Technical details and short-term outcomes. World J Gastroenterol. 2017;23:4293-302.

[6] Huang C-M, Zheng C-H, Li P, Xie J. Atlas of Laparoscopic Gastrectomy for Gastric Cancer. Springer Singapore. 2019.

[7] Huang C-M, Zheng C-H. Laparoscopic Gastrectomy for Gastric Cancer. Springer Netherlands. 2015.

[8] Kitano S, Yang H-K. Laparoscopic Gastrectomy for Cancer. Springer Japan. 2012.

[9] Ahn SH, Son SY, Lee CM, Jung DH, Park do J, Kim HH. Intracorporeal uncut Roux-en-Y gastrojejunostomy reconstruction in pure single-incision laparoscopic distal gastrectomy for early gastric cancer: unaided stapling closure. J Am Coll Surg. 2014;218:e17-21. 